\title{
Algebraic Equations with Span less than 4
}

\author{
By Raphael M. Robinson
}

1. Introduction. It is known [3] that an interval of length greater than 4 must contain infinitely many sets of conjugate algebraic integers, whereas an interval of length less than 4 can contain but a finite number of such sets. The problem remains open for intervals of length 4 , except when the end points are integers, in which case there are infinitely many sets of conjugates. For example, the interval $[-2,2]$ contains the numbers

$$
x=2 \cos 2 k \pi / m \quad[0 \leqq k \leqq m / 2, \quad(k, m)=1],
$$

which are a set of conjugate algebraic integers. Kronecker [1] showed that there are no other algebraic integers which lie with their conjugates in $[-2,2]$.

By the span of a polynomial $f(x)$ or of an algebraic equation $f(x)=0$ having only real roots, we mean the difference between the largest and smallest roots. We consider irreducible equations with integer coefficients and leading coefficient 1, so that the roots are a set of conjugate algebraic integers. Two such equations are considered equivalent if the roots of one can be obtained from the roots of the other by adding an integer, changing signs, or both. From what we have said, it follows that there are infinitely many inequivalent algebraic equations with span less than 4 , but, if $\epsilon>0$, only a finite number with span less than $4-\epsilon$.

Thus it appears that algebraic equations with span less than 4 are of particular interest. There are, of course, only a finite number of inequivalent equations of a given degree. We have attempted to make a list of representative equations, one chosen from each class of equivalent equations, with degrees from 2 to 8 . The representative may always be chosen in such a way that the average of the roots lies in $\left[0, \frac{1}{2}\right]$, and this restriction was accepted. If the average of the roots is 0 or $\frac{1}{2}$, then an ambiguity remains; the equation whose roots are in the shortest possible interval centered at $\frac{1}{4}$ was chosen. In $\$ 2$, we describe the method of computation which was used, and in $\S 3$, the results which were obtained.

2. Method of Computation. The computations described below were carried out during the period June-August 1961 on an IBM 704 at the Computer Center of the University of California, Berkeley. Single-precision floating point arithmetic (which corresponds to using 8 significant decimal digits) was used in the body of the computation. We had occasion to solve a great many algebraic equations, but in every case, these were equations all of whose roots were real. Newton's method was used throughout.

The problem is to find polynomials

$$
f(x)=x^{n}+a_{1} x^{n-1}+a_{2} x^{n-2}+\cdots+a_{n}
$$

with integer coefficients, having real roots and span less than 4 . It follows that all

Received December 13, 1963. This work was supported in part by the Miller Institute for Basic Research in Science. 
the derivatives $f^{\prime}(x), f^{\prime \prime}(x), \cdots$ also have real roots. Also, the span decreases, and hence remains less than 4 . Indeed, we can give [4] a better estimate for the span: The span of $f^{(j)}(x)$ for any such polynomial (at least for $n \leqq 25$ ) is not greater than in the special cases

$$
\begin{aligned}
& f(x)=(x-2)^{m}(x+2)^{m} \quad \text { if } n=2 m, \\
& f(x)=(x-2)^{m+1}(x+2)^{m} \quad \text { if } n=2 m+1 .
\end{aligned}
$$

Since we agreed that the average of the roots is to lie in $\left[0, \frac{1}{2}\right]$, we see that $-n / 2 \leqq a_{1} \leqq 0$. We try the values $a_{1}=0,-1,-2, \cdots$ in turn.

The general step in the computation consists in deciding what values should be allowed for $a_{k}$ after $a_{1}, a_{2}, \cdots, a_{k-1}$ have already been chosen. We assume that these coefficients were chosen so that the roots of $f^{(n-k+1)}(x)$ are real. Notice that $f^{(n-k)}(x)$ depends only on $a_{1}, a_{2}, \cdots, a_{k}$, and that a change in $a_{k}$ changes only the constant term of this polynomial. The values of $x$ which produce relative maxima and minima are the roots of $f^{(n-k+1)}(x)$, and are independent of $a_{k}$. Thus to make all the roots of $f^{(n-k)}(x)$ real, we need only insist that all of its relative maxima are positive and all of its relative minima are negative.

If $k \geqq 3$, this determines a certain interval in which $a_{k}$ can lie so that $f^{(n-k)}(x)$ will have real roots. (If $k=2$, we determine only an upper bound for $a_{k}$.) We can then test in each case whether the span of $f^{(n-k)}(x)$ is small enough. Specifically, the procedure chosen is as follows: We start $a_{k}$ at its largest possible value, and store its smallest value. In case $k<n$, then for each value of $a_{k}$ in turn, if the span of $f^{(n-k)}(x)$ is not too large, we increase $k$ by one unit and repeat the whole procedure.

If $k=n$, and the span of $f(x)$ is not too large, then we have succeeded in finding a polynomial of degree $n$ with span less than 4 . If this is irreducible, then it should be printed out. There was, however, a problem of what to do with the reducible polynomials. If they were all printed, the output would be too bulky. On the other hand, they would serve a useful purpose: We could look at them and see if all the expected ones were there, which would serve as a check on our work. As a compromise, some but not all of the reducible polynomials were printed. For example, for $n=6$ we printed those reducible sextics which are the product of two irreducible cubics.

If the span of $f^{(n-k)}(x)$ is too large, we can proceed to the next smaller value of $a_{k}$ without further calculation. After completing the calculation for the minimum possible value of $a_{k}$, we backtrack. That is, $k$ is reduced one unit, and we proceed to the next case at that level. If $k$ is even, a shortcut is possible. In this case, $f^{(n-k)}(x)$ is a polynomial of even degree $k$, so that as we decrease $a_{k}$ and therefore the constant term of the polynomial, the span increases. Thus as soon as we obtain too large a span, we can immediately backtrack. In case $k=2$, there is no smallest value of $a_{k}$ for which the roots of $f^{(n-k)}(x)$ are real, but we can still stop when the span gets too large.

The above procedure was carried out in complete detail for $n \leqq 6$. For degrees 7 and 8 , the time for the calculation became prohibitive. It was noticed that for $n \leqq 6$, the spans of $f^{(j)}(x)$ which actually occurred were somewhat smaller than the allowed maxima, and the values of the coefficients $a_{k}$ which actually led to solutions were in general rather near to the center of the allowed interval. Some reducible polynomials of degrees 7 and 8 were also examined. From all of these, guesses were 
made as to restrictions, both on the values of the coefficients and on the spans, which were stronger than those we could prove, but which were felt to be unlikely to exclude solutions. The calculations were carried out for $n=7$ and $n=8$ using these restrictions.

3. Discussion of the Results. The calculation described in $\$ 2$ produced a total of 96 inequivalent algebraic equations of degrees from 2 to 8 with span less than 4 . These were distributed as follows:

$\begin{array}{lllrrr}\text { Degree } & 2, \quad 3, \quad 4, \quad 5, \quad 6, \quad 7, & 8 . \\ \text { Equations } & 4, \quad 5, \quad 14, \quad 15, \quad 17, \quad 15, & 26 .\end{array}$

However, it should be kept in mind that we are not certain that the list is complete for degrees 7 and 8.

After these 96 equations were found, their roots were recomputed to double precision, and the discriminants of the equations were computed by multiplying the differences of the roots and squaring. In every case, the computed value of the discriminant differed from an integer by less than 0.001 .

The results of the computation are summarized in two tables. In Table 1, a list of the 96 equations is given, showing, in each case, the span, the gap (that is, the minimum distance between two roots), and the largest and smallest roots, all to four decimals, and finally the coefficients and the discriminant, with its factorization into primes. The equations are arranged first according to degree, and then by increasing span. The equations are identified by symbols $2 a, 2 b, \cdots, 8 z$, where the number is the degree, and the letter indicates the rank in the list by increasing span. In Table 2, all of the roots of the equations are given to ten decimals, the equations being identified by their symbols.

Of the 96 equations listed in Table 1, 19 correspond to Kronecker polynomials

$$
P_{m}(x)=\prod_{0 \leqq k \leqq m / 2 ;(k, m)=1}(x-2 \cos 2 k \pi / m)
$$

which have all their roots in $[-2,2]$. The equations, and the corresponding values of $m$, are as follows:

$$
\begin{aligned}
& \text { No. } 2 a, 2 b, 2 c, 3 a, 3 b, 4 c, 4 e, 4 f, 4 i, 5 a, 6 a, 6 g, 6 i, 6 k, 8 b, 8 e, 8 k, 8 p, 8 r . \\
& m=10,8,12,14,18,16,15,20,24,22,26,21,28,36,34,32,40,48,60 .
\end{aligned}
$$

Notice that if we change the sign of the roots of $P_{m}(x)$, then we double $m$ if $m$ is odd, halve $m$ if $m$ is twice an odd number, and leave $m$ unchanged if $m$ is a multiple of 4 . When $m=10$, it is also possible to diminish the roots by 1 , but this has the same effect as changing the signs. Thus the polynomials $P_{m}(x)$ not listed in Table 1 , but equivalent to entries there, are those with $m=5,7,9,11,13,17,30,42$. We should perhaps add that $P_{m}(x)$ has degree 1 when $m=1,2,3,4,6$, and degree 9 or greater in all cases which have not been mentioned.

It is known that the cyclotomic polynomial $Q_{m}(t)=t^{\phi(m)}+\cdots$, whose roots are the primitive $m$ th roots of 1 , has the discriminant

$$
(-1)^{\phi(m) / 2} m^{\phi(m)}: \prod_{p \mid m} p^{\phi(m) /(p-1)}
$$


TABLE 1

List of Algebraic Equations with Span Less than 4

\begin{tabular}{|c|c|c|c|}
\hline No. & $\begin{array}{l}\text { Span } \\
\text { Gap }\end{array}$ & $\begin{array}{l}\text { Max. Rt. } \\
\text { Min. Rt. }\end{array}$ & $\begin{array}{l}\text { Coefficients } \\
\text { Discriminant }\end{array}$ \\
\hline $2 a$ & $\begin{array}{l}2.2361 \\
2.2361\end{array}$ & $\begin{array}{r}1.6180 \\
-0.6180\end{array}$ & $\begin{array}{l}1-1-1 \\
5=\text { prime }\end{array}$ \\
\hline $2 b$ & $\begin{array}{l}2.8284 \\
2.8284\end{array}$ & $\begin{array}{r}1.4142 \\
-1.4142\end{array}$ & $\begin{array}{l}1+0-2 \\
8 \stackrel{0}{=} 2^{3}\end{array}$ \\
\hline $2 c$ & $\begin{array}{l}3.4641 \\
3.4641\end{array}$ & $\begin{array}{r}1.7321 \\
-1.7321\end{array}$ & $\begin{array}{l}1+0-3 \\
12 \stackrel{2^{2} \cdot 3}{3}\end{array}$ \\
\hline $2 d$ & $\begin{array}{l}3.6056 \\
3.6056\end{array}$ & $\begin{array}{r}2.3028 \\
-1.3028\end{array}$ & $\begin{aligned} 1 & -1-3 \\
13 & =\text { prime }\end{aligned}$ \\
\hline $3 a$ & $\begin{array}{l}3.0489 \\
1.3569\end{array}$ & $\begin{array}{r}1.8019 \\
-1.2470\end{array}$ & $\begin{array}{l}1-1-2+1 \\
49=7^{2}\end{array}$ \\
\hline $3 b$ & $\begin{array}{l}3.4115 \\
1.1848\end{array}$ & $\begin{array}{r}1.8794 \\
-1.5321\end{array}$ & $\begin{array}{c}1+0-3-1 \\
81=3^{4}\end{array}$ \\
\hline $3 c$ & $\begin{array}{l}3.6513 \\
1.7923\end{array}$ & $\begin{array}{r}2.1701 \\
-1.4812\end{array}$ & $\begin{array}{l}1-1-3+1 \\
148=2^{2} \cdot 37\end{array}$ \\
\hline $3 d$ & $\begin{array}{l}3.8895 \\
1.1359\end{array}$ & $\begin{array}{r}2.2143 \\
-1.6751\end{array}$ & $1+0-4-2$ \\
\hline $3 e$ & $\begin{array}{l}3.9757 \\
1.6067\end{array}$ & $\begin{array}{r}2.1149 \\
-1.8608 \\
\end{array}$ & $\begin{array}{c}1+0-4-1 \\
229=\text { prime }\end{array}$ \\
\hline $4 a$ & $\begin{array}{l}3.3871 \\
0.8986\end{array}$ & $\begin{array}{r}2.1935 \\
-1.1935\end{array}$ & $\begin{array}{c}1-2-2+3+1 \\
725=5^{2} \cdot 29\end{array}$ \\
\hline $4 b$ & $\begin{array}{l}3.4510 \\
0.8784\end{array}$ & $\begin{array}{r}2.0953 \\
-1.3557\end{array}$ & $\begin{array}{c}1-1-3+1+1 \\
725=5^{2} \cdot 29\end{array}$ \\
\hline $4 c$ & $\begin{array}{l}3.6955 \\
1.0824\end{array}$ & $\begin{array}{r}1.8478 \\
-1.8478\end{array}$ & $\begin{aligned} 1+0 & -4+0+2 \\
2048 & =2^{11}\end{aligned}$ \\
\hline $4 d$ & $\begin{array}{l}3.7032 \\
0.8289\end{array}$ & $\begin{array}{r}2.1268 \\
-1.5764\end{array}$ & $\begin{array}{c}1-1=4+2+3 \\
1957=19 \cdot 103\end{array}$ \\
\hline $4 e$ & $\begin{array}{l}3.7834 \\
0.4888\end{array}$ & $\begin{array}{r}1.8271 \\
-1.9563\end{array}$ & $\begin{array}{c}1-1=4+4+1 \\
1125=3^{2} \cdot 5^{3}\end{array}$ \\
\hline $4 f$ & $\begin{array}{l}3.8042 \\
0.7265\end{array}$ & $\begin{array}{r}1.9021 \\
-1.9021\end{array}$ & $\begin{array}{c}1+0=5+0+5 \\
2000=2^{4} \cdot 5^{3}\end{array}$ \\
\hline $4 g$ & $\begin{array}{l}3.8255 \\
1.0702\end{array}$ & $\begin{array}{r}2.0615 \\
-1.7640\end{array}$ & $\begin{aligned} 1+0 & =4-1+1 \\
1957 & =19 \cdot 103\end{aligned}$ \\
\hline $4 h$ & $\begin{array}{l}3.8592 \\
0.7474\end{array}$ & $\begin{array}{r}2.2631 \\
-1.5962\end{array}$ & $\begin{aligned} 1-2 & -3+5+1 \\
2777 & =\text { prime }\end{aligned}$ \\
\hline $4 i$ & $\begin{array}{l}3.8637 \\
1.0353\end{array}$ & $\begin{array}{r}1.9319 \\
-1.9319\end{array}$ & $\begin{array}{c}1+0-4+0+1 \\
2304=2^{8} \cdot 3^{2}\end{array}$ \\
\hline $4 j$ & $\begin{array}{l}3.8708 \\
0.8288\end{array}$ & $\begin{array}{r}2.3623 \\
-1.5085\end{array}$ & $\begin{aligned} 1-1 & -4+1+2 \\
2777 & =\text { prime }\end{aligned}$ \\
\hline $4 k$ & $\begin{array}{l}3.9279 \\
0.5657\end{array}$ & $\begin{array}{r}2.1439 \\
-1.7840\end{array}$ & $\begin{aligned} 1+0 & -5-1+4 \\
2777 & =\text { prime }\end{aligned}$ \\
\hline $4 l$ & $\begin{array}{l}3.9335 \\
0.6892 \\
\end{array}$ & $\begin{array}{r}2.4667 \\
-1.4667 \\
\end{array}$ & $\begin{array}{c}1-2-4+5+5 \\
2525=5^{2} \cdot 101\end{array}$ \\
\hline
\end{tabular}


TABLE 1-Continued

\begin{tabular}{|c|c|c|c|}
\hline No. & $\begin{array}{l}\text { Span } \\
\text { Gap }\end{array}$ & $\begin{array}{l}\text { Max. Rt. } \\
\text { Min. Rt. }\end{array}$ & $\begin{array}{l}\text { Coefficients } \\
\text { Discriminant }\end{array}$ \\
\hline $4 m$ & $\begin{array}{l}3.9644 \\
0.8744\end{array}$ & $\begin{array}{r}2.5231 \\
-1.4413\end{array}$ & $\begin{aligned} 1-1 & -4+0+1 \\
1957 & =19 \cdot 103\end{aligned}$ \\
\hline $4 n$ & $\begin{array}{l}3.9910 \\
1.2758\end{array}$ & $\begin{array}{r}2.4955 \\
-1.4955\end{array}$ & $\begin{array}{c}1-2-3+4+1 \\
4752=2^{4} \cdot 3^{3} \cdot 11\end{array}$ \\
\hline $5 a$ & $\begin{array}{l}3.6015 \\
0.6093\end{array}$ & $\begin{array}{r}1.9190 \\
-1.6825\end{array}$ & $\begin{array}{r}1-1-4+3+3-1 \\
14641=11^{4}\end{array}$ \\
\hline $5 b$ & $\begin{array}{l}3.7396 \\
0.4197\end{array}$ & $\begin{array}{r}2.0431 \\
-1.6965\end{array}$ & $\begin{aligned} 1+0-5 & =1+5+1 \\
24217 & =61 \cdot 397\end{aligned}$ \\
\hline $5 c$ & $\begin{array}{l}3.7645 \\
0.4931\end{array}$ & $\begin{array}{r}2.1064 \\
-1.6582\end{array}$ & $\begin{aligned} 1-1-5 & +3+6-1 \\
36497 & =\text { prime }\end{aligned}$ \\
\hline $5 d$ & $\begin{array}{l}3.7760 \\
0.4333\end{array}$ & $\begin{array}{r}2.2242 \\
-1.5518\end{array}$ & $\begin{aligned} 1-2-4 & +7+4-5 \\
24217 & =61 \cdot 397\end{aligned}$ \\
\hline $5 e$ & $\begin{array}{l}3.7852 \\
0.6208\end{array}$ & $\begin{array}{r}2.4498 \\
-1.3354\end{array}$ & $\begin{array}{r}1-2-3+4+2-1 \\
24217=61 \cdot 397\end{array}$ \\
\hline $5 f$ & $\begin{array}{l}3.8439 \\
0.6789\end{array}$ & $\begin{array}{r}2.1388 \\
-1.7050\end{array}$ & $\begin{aligned} 1-2-3 & +6+0-1 \\
24217 & =61 \cdot 397\end{aligned}$ \\
\hline $5 g$ & $\begin{array}{l}3.8743 \\
0.9167\end{array}$ & $\begin{array}{r}2.3180 \\
-1.5563\end{array}$ & $\begin{aligned} 1-\underset{36}{2}-3 & +5+1-1 \\
497 & =\text { prime }\end{aligned}$ \\
\hline $5 h$ & $\begin{array}{l}3.9130 \\
0.4177\end{array}$ & $\begin{array}{r}1.8866 \\
-2.0264\end{array}$ & $\begin{array}{rl}1-1-5 & +5+4-3 \\
36 & 497=\text { prime }\end{array}$ \\
\hline $5 i$ & $\begin{array}{l}3.9222 \\
0.7285\end{array}$ & $\begin{array}{r}2.0851 \\
-1.8371\end{array}$ & $\begin{aligned} 1-1-5 & +4+5-3 \\
65657 & =\text { prime }\end{aligned}$ \\
\hline $5 j$ & $\begin{array}{l}3.9469 \\
0.3327\end{array}$ & $\begin{array}{r}2.4039 \\
-1.5430\end{array}$ & $\begin{array}{c}1-1-5+2+5-1 \\
38569=\text { prime }\end{array}$ \\
\hline $5 k$ & $\begin{array}{l}3.9635 \\
0.4993\end{array}$ & $\begin{array}{r}2.3283 \\
-1.6353\end{array}$ & $\begin{aligned} 1-2-4 & +6+4-1 \\
81 & +509=\text { prime }\end{aligned}$ \\
\hline $5 l$ & $\begin{array}{l}3.9757 \\
0.3711\end{array}$ & $\begin{array}{r}2.0541 \\
-1.9216\end{array}$ & $\begin{aligned} 1+0-6 & +0+8-1 \\
81589 & =83.983\end{aligned}$ \\
\hline $5 m$ & $\begin{array}{l}3.9797 \\
0.6950\end{array}$ & $\begin{array}{r}2.3928 \\
-1.5869\end{array}$ & $\begin{array}{c}1-2-4+6+4-2 \\
126032=2^{4} \cdot 7877\end{array}$ \\
\hline $5 n$ & $\begin{array}{l}3.9821 \\
0.5726\end{array}$ & $\begin{array}{r}2.2684 \\
-1.7138\end{array}$ & $\begin{aligned} 1-1-5 & +3+5-2 \\
81 & +509=\text { prime }\end{aligned}$ \\
\hline $5 o$ & $\begin{array}{l}3.9926 \\
0.5149\end{array}$ & $\begin{array}{r}2.0385 \\
-1.9541\end{array}$ & $\begin{aligned} 1+0-5 & +0+4-1 \\
38569 & \stackrel{0}{=} \text { prime }\end{aligned}$ \\
\hline $6 a$ & $\begin{array}{l}3.7128 \\
0.4449\end{array}$ & $\begin{array}{r}1.9419 \\
-1.7709\end{array}$ & $\begin{array}{c}1-1-5+4+6-3-1 \\
371293=13^{5}\end{array}$ \\
\hline $6 b$ & $\begin{array}{l}3.7400 \\
0.5265\end{array}$ & $\begin{array}{r}2.3700 \\
-1.3700\end{array}$ & $1-3-2+9+\underset{434}{2}+\frac{1}{=}+1-6-1$ \\
\hline $6 c$ & $\begin{array}{l}3.7970 \\
0.5006\end{array}$ & $\begin{array}{r}2.2831 \\
-1.5140\end{array}$ & $\begin{array}{c}1-3-2+10-1-1-7+1 \\
485125=5^{3} \cdot 3881\end{array}$ \\
\hline $6 d$ & $\begin{array}{l}3.8077 \\
0.3869\end{array}$ & $\begin{array}{r}2.1588 \\
-1.6490\end{array}$ & $\begin{array}{c}1-2-4+7+4-4-1 \\
592661=\text { prime }\end{array}$ \\
\hline
\end{tabular}


TABLE 1-Continued

\begin{tabular}{|c|c|c|c|}
\hline No. & $\begin{array}{l}\text { Span } \\
\text { Gap }\end{array}$ & $\begin{array}{l}\text { Max. Rt. } \\
\text { Min. Rt. }\end{array}$ & $\begin{array}{l}\text { Coefficients } \\
\text { Discriminant }\end{array}$ \\
\hline $6 e$ & $\begin{array}{l}3.8633 \\
0.5392\end{array}$ & $\begin{array}{r}2.4316 \\
-1.4316\end{array}$ & $\begin{array}{c}1-3-2+2+9+\underset{810448}{=}+2^{4} \cdot 37^{3}\end{array}$ \\
\hline $6 f$ & $\begin{array}{l}3.8865 \\
0.3177\end{array}$ & $\begin{array}{r}2.0702 \\
-1.8163\end{array}$ & $\begin{array}{r}1-1-6+5+\underset{1202933}{=}+79 \cdot 15227-1 \\
\quad-6-1\end{array}$ \\
\hline $6 g$ & $\begin{array}{l}3.8888 \\
0.2587\end{array}$ & $\begin{array}{r}1.9111 \\
-1.9777\end{array}$ & $\begin{array}{c}1-1-6+6+8-8-8+1 \\
453789=3^{3} \cdot 7^{5}\end{array}$ \\
\hline $6 h$ & $\begin{array}{l}3.8895 \\
0.3331\end{array}$ & $\begin{array}{r}2.4448 \\
-1.4448\end{array}$ & $\begin{array}{c}1-3-3+11+3-9-1 \\
1528713=3^{8} \cdot 233\end{array}$ \\
\hline $6 i$ & $\begin{array}{l}3.8997 \\
0.3862\end{array}$ & $\begin{array}{r}1.9499 \\
-1.9499\end{array}$ & $\begin{array}{l}1+\underset{1075}{0-7+048}=2^{6} \cdot 7^{5} \\
\quad+14+0-7\end{array}$ \\
\hline $6 j$ & $\begin{array}{l}3.9293 \\
0.4378\end{array}$ & $\begin{array}{r}2.1233 \\
-1.8060\end{array}$ & $\begin{array}{c}1-2-4+8+2-5+1 \\
485125=5^{3} \cdot 3881\end{array}$ \\
\hline $6 k$ & $\begin{array}{l}3.9392 \\
0.6015\end{array}$ & $\begin{array}{r}1.9696 \\
-1.9696\end{array}$ & $\begin{array}{c}1+\underset{1259712=2^{6} \cdot 3^{9}}{0}+0-3\end{array}$ \\
\hline $6 l$ & $\begin{array}{l}3.9427 \\
0.3024\end{array}$ & $\begin{array}{r}2.3736 \\
-1.5691\end{array}$ & $\begin{array}{c}1-2-5+7+8-3-1 \\
1241125=5^{3.9929}\end{array}$ \\
\hline $6 m$ & $\begin{array}{l}3.9456 \\
0.5345\end{array}$ & $\begin{array}{r}2.0467 \\
-1.8989\end{array}$ & $1-1-5+5+4+5-2-1$ \\
\hline $6 n$ & $\begin{array}{l}3.9457 \\
0.3125\end{array}$ & $\begin{array}{r}2.2552 \\
-1.6906\end{array}$ & $\begin{array}{c}1-2-5+9+6-9+1 \\
1416125=5^{3} \cdot 11329\end{array}$ \\
\hline $6 o$ & $\begin{array}{l}3.9507 \\
0.4485\end{array}$ & $\begin{array}{r}2.3448 \\
-1.6059\end{array}$ & $\begin{array}{c}1-2-5+8+8-7-4 \\
1868969=107 \cdot 17467\end{array}$ \\
\hline $6 p$ & $\begin{array}{l}3.9979 \\
0.4211\end{array}$ & $\begin{array}{r}2.2050 \\
-1.7929\end{array}$ & $\begin{array}{l}1-1-6+4+9-3-3-1 \\
3086597=383 \cdot 8059\end{array}$ \\
\hline $6 q$ & $\begin{array}{l}3.9986 \\
0.2324\end{array}$ & $\begin{array}{r}2.4249 \\
-1.5737\end{array}$ & $\begin{array}{l}1-2-5+8+7-7-1 \\
2286997=349 \cdot 6553\end{array}$ \\
\hline $7 a$ & $\begin{array}{l}3.8630 \\
0.3407\end{array}$ & $\begin{array}{r}2.2788 \\
-1.5842\end{array}$ & $\begin{array}{c}1-2-5+8+8+8-7-7-3+1 \\
20134393=71 \cdot 283583\end{array}$ \\
\hline $7 b$ & $\begin{array}{l}3.8646 \\
0.3444\end{array}$ & $\begin{array}{r}2.3912 \\
-1.4733\end{array}$ & $\begin{aligned} 1-3-3+12+2-13+0 & -3+3 \\
& 25164057=3 \cdot 8388019\end{aligned}$ \\
\hline $7 c$ & $\begin{array}{l}3.8809 \\
0.2639\end{array}$ & $\begin{array}{r}2.0889 \\
-1.7921\end{array}$ & $1-\frac{1-7+5+15-6-9+1}{41153941=\text { prime }}$ \\
\hline $7 d$ & $\begin{array}{l}3.8853 \\
0.3173\end{array}$ & $\begin{array}{r}2.2412 \\
-1.6441\end{array}$ & $1-\underset{25164057=3 \cdot 8-5388019}{2-5+9}=$ \\
\hline $7 e$ & $\begin{array}{l}3.9022 \\
0.3001\end{array}$ & $\begin{array}{r}2.1532 \\
-1.7490\end{array}$ & $1-\underset{28118}{2-569=\text { prime }}$ \\
\hline $7 f$ & $\begin{array}{l}3.9243 \\
0.4317\end{array}$ & $\begin{array}{r}2.2018 \\
-1.7225\end{array}$ & $\begin{array}{c}1-1-6+4+10-4-4-4+1 \\
20134393=71 \cdot 283583\end{array}$ \\
\hline $7 g$ & $\begin{array}{l}3.9251 \\
0.2437\end{array}$ & $\begin{array}{r}2.5366 \\
-1.3885\end{array}$ & $\begin{aligned} 1- & 3-3+11+3-10-1+1 \\
& 32354821=\text { prime }\end{aligned}$ \\
\hline $7 h$ & $\begin{array}{l}3.9323 \\
0.2532\end{array}$ & $\begin{array}{r}2.1867 \\
-1.7456\end{array}$ & $1-\frac{1-7+4+15-2-8-8-1}{43242544=2^{4} \cdot 101 \cdot 26759}$ \\
\hline $7 i$ & $\begin{array}{l}3.9373 \\
0.4349\end{array}$ & $\begin{array}{r}2.2756 \\
-1.6617\end{array}$ & $\begin{array}{c}1-3-3+13+0-14+2+3 \\
30653489=1399 \cdot 21911\end{array}$ \\
\hline
\end{tabular}


TABLE 1-Continued

\begin{tabular}{|c|c|c|c|}
\hline No. & $\begin{array}{l}\text { Span } \\
\text { Gap }\end{array}$ & $\begin{array}{l}\text { Max. Rt. } \\
\text { Min. Rt. }\end{array}$ & $\begin{array}{l}\text { Coefficients } \\
\text { Discriminant }\end{array}$ \\
\hline $7 j$ & $\begin{array}{l}3.9600 \\
0.3119\end{array}$ & $\begin{array}{r}2.0768 \\
-1.8832\end{array}$ & $\begin{array}{rl}1- & 1-7+5+15-5-10-1 \\
39829 & 513=373 \cdot 106781\end{array}$ \\
\hline $7 k$ & $\begin{array}{l}3.9704 \\
0.1028\end{array}$ & $\begin{array}{r}2.0341 \\
-1.9363\end{array}$ & $\begin{array}{c}1+\underset{34}{0}-854953=0+19+0-12-1 \\
+ \text { prime }\end{array}$ \\
\hline $7 l$ & $\begin{array}{l}3.9713 \\
0.3383\end{array}$ & $\begin{array}{r}2.2101 \\
-1.7612\end{array}$ & $\begin{array}{c}1-2-6+11+11-17-6+7 \\
41455873=37 \cdot 1120429\end{array}$ \\
\hline $7 m$ & $\begin{array}{l}3.9754 \\
0.1724\end{array}$ & $\begin{array}{r}2.3144 \\
-1.6610\end{array}$ & $\begin{aligned} 1-1-7+4+15- & -4-9-1 \\
& 35269513=\text { prime }\end{aligned}$ \\
\hline $7 n$ & $\begin{array}{l}3.9824 \\
0.2364\end{array}$ & $\begin{array}{r}2.0780 \\
-1.9043\end{array}$ & $\begin{array}{c}1-1-7+6+13-9-3+1 \\
39610073=89 \cdot 599 \cdot 743\end{array}$ \\
\hline 70 & $\begin{array}{l}3.9980 \\
0.3251 \\
\end{array}$ & $\begin{array}{r}2.4775 \\
-1.5206 \\
\end{array}$ & $\begin{array}{r}1-3-3+11+3-9-2+1 \\
32567681=89 \cdot 365929\end{array}$ \\
\hline $8 a$ & $\begin{array}{l}3.7977 \\
0.2474\end{array}$ & $\begin{array}{r}2.3989 \\
-1.3989\end{array}$ & $\begin{array}{c}1-4-1+17-5-23+6+9-1 \\
309593125=5^{4} \cdot 19 \cdot 29^{2} \cdot 31\end{array}$ \\
\hline $8 b$ & $\begin{array}{l}3.8309 \\
0.2655\end{array}$ & $\begin{array}{r}1.9659 \\
-1.8649\end{array}$ & $1-1-\underset{410}{1}+6+638673=15=17^{7}-10+4+1$ \\
\hline $8 c$ & $\begin{array}{l}3.9122 \\
0.2248\end{array}$ & $\begin{array}{r}2.4561 \\
-1.4561\end{array}$ & $\begin{array}{r}1-4-1+17-6-6-21+8+6-1 \\
1459+172+469=3 \cdot 19^{2} \cdot 103^{2} \cdot 127\end{array}$ \\
\hline $8 d$ & $\begin{array}{l}3.9186 \\
0.2723\end{array}$ & $\begin{array}{r}2.4288 \\
-1.4898\end{array}$ & $\begin{array}{r}1-3-4+14+6-6-19-5+6+1 \\
1348097653=7^{2} \cdot 27512197\end{array}$ \\
\hline $8 e$ & $\begin{array}{l}3.9231 \\
0.2986\end{array}$ & $\begin{array}{r}1.9616 \\
-1.9616\end{array}$ & $\begin{array}{c}1+0-8+0+20+0-16+0+2 \\
2147483648=2^{31}\end{array}$ \\
\hline $8 f$ & $\begin{array}{l}3.9294 \\
0.2923\end{array}$ & $\begin{array}{r}2.0745 \\
-1.8549\end{array}$ & $\begin{array}{c}1+0-8-1+20+4-16-3+2 \\
1359341129=\text { prime }\end{array}$ \\
\hline $8 g$ & $\begin{array}{l}3.9337 \\
0.2709\end{array}$ & $\begin{array}{r}2.3339 \\
-1.5998\end{array}$ & $\begin{array}{r}1-3-4+15+4-22+0+9+1 \\
1391339501=71 \cdot 149 \cdot 131519\end{array}$ \\
\hline $8 h$ & $\begin{array}{l}3.9380 \\
0.2334\end{array}$ & $\begin{array}{r}2.3788 \\
-1.5593\end{array}$ & $\begin{array}{c}1-3-4+15+4-22+0+8-1 \\
\begin{array}{c}1299600812 \\
=\end{array}=2^{2} \cdot 324900203\end{array}$ \\
\hline $8 i$ & $\begin{array}{l}3.9415 \\
0.2393\end{array}$ & $\begin{array}{r}2.2321 \\
-1.7094\end{array}$ & $\begin{array}{r}1-2-6+11+11-17-6+6+1 \\
1348097653=7^{2} \cdot 27512197\end{array}$ \\
\hline $8 j$ & $\begin{array}{l}3.9465 \\
0.2745\end{array}$ & $\begin{array}{r}2.2893 \\
-1.6573\end{array}$ & $\begin{array}{c}1-2-6+10+12-13-8+3+1 \\
1299600812=2^{2} \cdot 324900203\end{array}$ \\
\hline $8 k$ & $\begin{array}{l}3.9508 \\
0.1934\end{array}$ & $\begin{array}{r}1.9754 \\
-1.9754\end{array}$ & 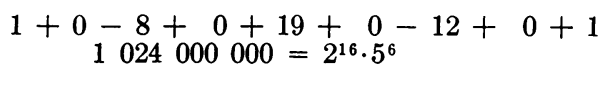 \\
\hline $8 l$ & $\begin{array}{l}3.9531 \\
0.1320\end{array}$ & $\begin{array}{r}2.3871 \\
-1.5661\end{array}$ & $\begin{array}{c}1-4-2+21-6-33+12+13-1 \\
1077044573=11 \cdot 97913143\end{array}$ \\
\hline $8 m$ & $\begin{array}{l}3.9537 \\
0.0856\end{array}$ & $\begin{array}{r}2.2356 \\
-1.7181\end{array}$ & $\begin{array}{c}1-3-\underset{707}{5}+18+7-33-3+3+18+1 \\
=9973 \cdot 70 \\
921\end{array}$ \\
\hline $8 n$ & $\begin{array}{l}3.9627 \\
0.2508\end{array}$ & $\begin{array}{r}2.1504 \\
-1.8123\end{array}$ & $\begin{array}{r}1-2-6+11+11-16-7+5+1 \\
1621897976=2^{3} \cdot 239 \cdot 848273\end{array}$ \\
\hline 80 & $\begin{array}{l}3.9649 \\
0.2369\end{array}$ & $\begin{array}{r}2.2215 \\
-1.7434\end{array}$ & $\begin{array}{c}1-1-7+5+15-7-10+2+1 \\
661518125=5^{4} \cdot 439 \cdot 2411\end{array}$ \\
\hline
\end{tabular}


TABLE 1-Continued

\begin{tabular}{|c|c|c|c|c|}
\hline No. & $\begin{array}{l}\text { Span } \\
\text { Gap }\end{array}$ & $\begin{array}{l}\text { Max. Rt. } \\
\text { Min. Rt. }\end{array}$ & $\begin{array}{l}\text { Coefficients } \\
\text { Discriminant }\end{array}$ & \\
\hline $8 p$ & $\begin{array}{l}3.9658 \\
0.3692\end{array}$ & $\begin{array}{r}1.9829 \\
-1.9829\end{array}$ & $\begin{array}{r}1+0-8+0+20+\underset{1358}{854}+0-16+ \\
496=2^{24} \cdot 3^{4}\end{array}$ & $0+1$ \\
\hline $8 q$ & $\begin{array}{l}3.9777 \\
0.2130\end{array}$ & $\begin{array}{r}2.2921 \\
-1.6857\end{array}$ & $\begin{array}{r}1-3-4+15+4-21-2+ \\
1442599461=3^{2} \cdot 29 \cdot 2351^{2}\end{array}$ & $8+1$ \\
\hline $8 r$ & $\begin{array}{l}3.9781 \\
0.3976\end{array}$ & $\begin{array}{r}1.9890 \\
-1.9890\end{array}$ & $\begin{array}{r}1+0-7+0+14+0-8-8+ \\
324000000=2^{8} \cdot 3^{4} \cdot 5^{6}\end{array}$ & $0+1$ \\
\hline $8 s$ & $\begin{array}{l}3.9815 \\
0.4274\end{array}$ & $\begin{array}{r}2.1461 \\
-1.8354\end{array}$ & $\begin{array}{r}1-1-7+5+15-6-10+ \\
1424875717=15493 \cdot 91969\end{array}$ & $1+1$ \\
\hline $8 t$ & $\begin{array}{l}3.9827 \\
0.2028\end{array}$ & $\begin{array}{r}2.2589 \\
-1.7238\end{array}$ & $\begin{array}{r}1-2-7+12+17-21-16+ \\
2121175625=5^{4} \cdot 3393881\end{array}$ & $10+5$ \\
\hline $8 u$ & $\begin{array}{l}3.9841 \\
0.2001\end{array}$ & $\begin{array}{r}2.2753 \\
-1.7088\end{array}$ & $\begin{array}{r}1-2-7+11+18-15-18+ \\
1077044573=11.97913143\end{array}$ & $0+1$ \\
\hline $8 v$ & $\begin{array}{l}3.9858 \\
0.2397\end{array}$ & $\begin{array}{r}2.2853 \\
-1.7005\end{array}$ & $\begin{array}{c}1-2-7+12+17 \underset{2-21}{2} 256866749=15+ \\
=\text { prime }\end{array}$ & $9+1$ \\
\hline $8 w$ & $\begin{array}{l}3.9865 \\
0.2625\end{array}$ & $\begin{array}{r}2.2306 \\
-1.7559\end{array}$ & $1-1_{1-8}+\underset{549}{5} 23+21-6-6-18+$ & $2+3$ \\
\hline $8 x$ & $\begin{array}{l}3.9893 \\
0.2510\end{array}$ & $\begin{array}{r}2.3816 \\
-1.6078\end{array}$ & $\begin{array}{r}1-4-1+18-9-21+13+ \\
1994682269=1877 \cdot 1062697\end{array}$ & $3-1$ \\
\hline $8 y$ & $\begin{array}{l}3.9955 \\
0.2647\end{array}$ & $\begin{array}{r}2.2974 \\
-1.6981\end{array}$ & $\begin{array}{c}1-1-7+4+15-3-9+ \\
483345053=\text { prime }\end{array}$ & $0+1$ \\
\hline $8 z$ & $\begin{array}{l}3.9973 \\
0.1868\end{array}$ & $\begin{array}{r}2.3517 \\
-1.6456\end{array}$ & 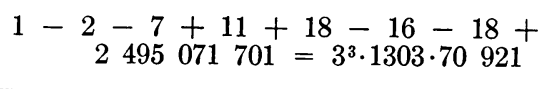 & $3+3$ \\
\hline
\end{tabular}

for $m>2$, where $p$ runs through the prime divisors of $m$. A proof may be found in E. Lehmer [2]. Since $Q_{m}(t)=t^{\phi(m) / 2} P_{m}(t+1 / t)$ for $m>2$, this result can be used to find the discriminant of $P_{m}(x)$. Indeed, it is easily seen that the discriminant of $P_{m}(x)$ for $m>2$ is equal to

$$
\left(m^{\phi(m)}: c \prod_{p \mid m} p^{\phi(m) /(p-1)}\right)^{1 / 2}
$$

where

$$
c=\left\{\begin{array}{l}
p \text { if } m=p^{l}, 2 p^{l} \quad(p>2) \\
4 \text { if } m=2^{l} \\
1 \text { otherwise. }
\end{array}\right.
$$

Here $p$ denotes a prime, and $l$ a positive integer. The computed values agree with those found from this formula. The formula shows that the discriminants of the Kronecker polynomials are very round numbers. A glance at Table 1 shows that most of the other discriminants are not so round; indeed, many of them are prime.

Another observation is that many of the discriminants occur repeatedly. For example, there are four different quintics with discriminant 24217.

An interesting fact that does not show in Table 1, but was apparent in the preliminary output, is that there is a strong correlation between the smallness of the gap between the nearest roots of a polynomial, and the reducibility of the poly- 
TABle 2

Roots of Algebraic Equations with Span less than 4

\begin{tabular}{|c|c|c|c|c|c|}
\hline $\begin{array}{r}2 a \\
1.61803 \\
-0.61803 \\
\end{array}$ & $\begin{array}{l}39887 \\
39887 \\
\end{array}$ & $\begin{array}{r}2 b \\
1.4142135624 \\
-1.4142135624 \\
\end{array}$ & $\begin{array}{r}2 c \\
1.73205 \\
-1.73205 \\
\end{array}$ & $\begin{array}{l}08076 \\
08076 \\
\end{array}$ & $\begin{array}{r}2 d \\
2.3027756377 \\
-1.3027756377 \\
\end{array}$ \\
\hline $\begin{array}{r}3 a \\
1.80193 \\
0.44504 \\
-1.24697\end{array}$ & $\begin{array}{l}77358 \\
18679 \\
96037\end{array}$ & $\begin{array}{r}3 b \\
1.8793852416 \\
-0.3472963553 \\
-1.5320888862\end{array}$ & $\begin{array}{r}3 c \\
2.17008 \\
0.31110 \\
-1.48119\end{array}$ & $\begin{array}{l}64866 \\
78175 \\
43041\end{array}$ & $\begin{array}{r}3 d \\
2.2143197434 \\
-0.5391888728 \\
-1.6751308706\end{array}$ \\
\hline $\begin{array}{r}3 e \\
2.11490 \\
-0.25410 \\
-1.86080\end{array}$ & $\begin{array}{l}75415 \\
16884 \\
58531\end{array}$ & 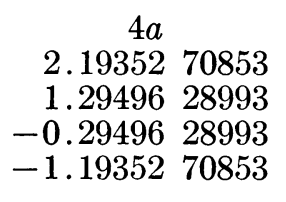 & $\begin{array}{r}4 b \\
2.09529 \\
0.73764 \\
-0.47725 \\
-1.35567\end{array}$ & $\begin{array}{l}39852 \\
03052 \\
99965 \\
42940\end{array}$ & $\begin{array}{rr}4 c & \\
1.84775 & 90650 \\
0.76536 & 68647 \\
-0.76536 & 68647 \\
-1.84775 & 90650\end{array}$ \\
\hline $\begin{array}{r}4 d \\
2.12675 \\
1.19712 \\
-0.74746 \\
-1.57641\end{array}$ & $\begin{array}{l}70596 \\
62976 \\
84539 \\
49033\end{array}$ & $\begin{array}{rr}4 e & \\
1.82709 & 09153 \\
1.33826 & 12127 \\
-0.20905 & 69265 \\
-1.95629 & 52015\end{array}$ & $\begin{array}{r}4 f \\
1.90211 \\
1.17557 \\
-1.17557 \\
-1.90211\end{array}$ & $\begin{array}{l}30326 \\
05046 \\
05046 \\
30326\end{array}$ & $\begin{array}{r}4 g \\
2.0614988507 \\
0.3963385310 \\
-0.6938224565 \\
-1.7640149252\end{array}$ \\
\hline $\begin{array}{r}4 h \\
2.26307 \\
1.51572 \\
-0.18264 \\
-1.59615\end{array}$ & $\begin{array}{l}74103 \\
15893 \\
43236 \\
46760\end{array}$ & $\begin{array}{rr}4 i \\
1.93185 & 16526 \\
0.51763 & 80902 \\
-0.51763 & 80902 \\
-1.93185 & 16526\end{array}$ & $\begin{array}{r}4 j \\
2.36233 \\
0.82578 \\
-0.67964 \\
-1.50848 \\
\end{array}$ & $\begin{array}{l}98329 \\
45519 \\
31856 \\
11992 \\
\end{array}$ & $\begin{array}{rr}4 k \\
2.14386 & 44258 \\
0.85844 & 19548 \\
-1.21830 & 96530 \\
-1.78399 & 67275\end{array}$ \\
\hline $\begin{array}{r}4 l \\
2.46673 \\
1.77748 \\
-0.77748 \\
-1.46673\end{array}$ & $\begin{array}{l}18040 \\
42509 \\
42509 \\
18040\end{array}$ & 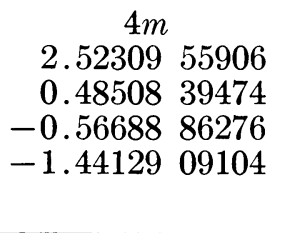 & $\begin{array}{r}4 n \\
2.49550 \\
1.21968 \\
-0.21968 \\
-1.49550\end{array}$ & $\begin{array}{l}76 \\
68 \\
68 \\
76\end{array}$ & \begin{tabular}{rr}
\multicolumn{2}{c}{$5 a$} \\
1.91898 & 59472 \\
1.30972 & 14679 \\
0.28462 & 96765 \\
-0.83083 & 00260 \\
-1.68250 & 70657
\end{tabular} \\
\hline $\begin{array}{r}5 b \\
2.04314 \\
1.13012 \\
-0.19993 \\
-1.27683 \\
-1.69649 \\
\end{array}$ & $\begin{array}{l}09156 \\
71160 \\
34460 \\
96986 \\
48871\end{array}$ & $\begin{array}{r}5 c \\
2.1063697048 \\
1.5592959532 \\
0.1575967325 \\
-1.1650943780 \\
-1.6581680124 \\
\end{array}$ & $\begin{array}{r}5 d \\
2.22418 \\
1.67128 \\
0.77490 \\
-1.11854 \\
-1.55183 \\
\end{array}$ & $\begin{array}{l}46483 \\
35260 \\
94377 \\
26729 \\
49391\end{array}$ & \begin{tabular}{rr}
\multicolumn{2}{c}{$5 e$} \\
2.44982 & 94641 \\
1.26073 & 41678 \\
0.33932 & 84354 \\
-0.71453 & 30635 \\
-1.33535 & 90038 \\
\end{tabular} \\
\hline $\begin{array}{r}5 f \\
2.13882 \\
1.45989 \\
0.48980 \\
-0.38348 \\
-1.70503\end{array}$ & $\begin{array}{l}90696 \\
17376 \\
28469 \\
42661 \\
93880\end{array}$ & $\begin{array}{rr}5 g \\
2.31801 & 35147 \\
1.33418 & 51529 \\
0.41037 & 53841 \\
-0.50628 & 72600 \\
-1.55628 & 67917\end{array}$ & $\begin{array}{r}5 h \\
1.88660 \\
1.46887 \\
0.58466 \\
-0.91373 \\
-2.02641\end{array}$ & $\begin{array}{l}96452 \\
60099 \\
39955 \\
17013 \\
79492\end{array}$ & $\begin{array}{rr}5 i \\
2.08508 & 11918 \\
1.32892 & 33446 \\
0.53163 & 32638 \\
-1.10855 & 64842 \\
-1.83708 & 13160\end{array}$ \\
\hline $\begin{array}{r}5 j \\
2.40387 \\
1.15694 \\
0.19253 \\
-1.21033 \\
-1.54301\end{array}$ & $\begin{array}{l}48019 \\
20520 \\
13863 \\
19657 \\
62745\end{array}$ & 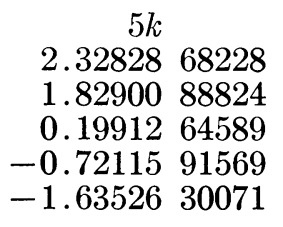 & $\begin{array}{r}5 l \\
2.05410 \\
1.29150 \\
0.12651 \\
-1.55052 \\
-1.92159\end{array}$ & $\begin{array}{l}71518 \\
07115 \\
46910 \\
65930 \\
59613\end{array}$ & $\begin{array}{rr}5 m \\
2.39275 & 62472 \\
1.69774 & 65488 \\
0.35947 & 02421 \\
-0.86306 & 70539 \\
-1.58690 & 59843\end{array}$ \\
\hline
\end{tabular}


TABLE 2-Continued

\begin{tabular}{|c|c|c|c|}
\hline \begin{tabular}{rr}
\multicolumn{2}{c}{$5 n$} \\
2.26835 & 33165 \\
1.21567 & 55100 \\
0.37086 & 49091 \\
-1.14112 & 85272 \\
-1.71376 & 52084
\end{tabular} & \begin{tabular}{rr}
\multicolumn{2}{c}{$5 o$} \\
2.03849 & 52910 \\
0.79073 & 43035 \\
0.27583 & 41933 \\
-1.15098 & 41733 \\
-1.95407 & 96146
\end{tabular} & $\begin{array}{rr}6 a \\
1.9418836349 \\
1.49702 & 14963 \\
0.70920 & 97741 \\
-0.24107 & 33605 \\
-1.13612 & 94935 \\
-1.77091 & 20513\end{array}$ & \begin{tabular}{rr}
\multicolumn{2}{c}{$6 b$} \\
2.37002 & 12843 \\
1.84348 & 73025 \\
1.16937 & 45321 \\
-0.16937 & 45321 \\
-0.84348 & 73025 \\
-1.37002 & 12843
\end{tabular} \\
\hline $\begin{array}{rr}6 c \\
2.28306 & 70725 \\
1.75190 & 56915 \\
1.25135 & 09117 \\
0.14401 & 29992 \\
-0.91638 & 39954 \\
-1.51395 & 26794\end{array}$ & $\begin{array}{rr}6 d \\
2.15875 & 16408 \\
1.77183 & 58725 \\
0.81582 & 30347 \\
-0.22199 & 32171 \\
-0.87542 & 74923 \\
-1.64898 & 98386\end{array}$ & $\begin{array}{rr}6 e \\
2.4316299604 \\
1.89244 & 10876 \\
0.78268 & 97829 \\
0.21731 & 02171 \\
-0.89244 & 10876 \\
-1.43162 & 99604\end{array}$ & $\begin{array}{rr}6 f \\
2.0701594250 \\
1.5840897733 \\
0.80055 & 70644 \\
-0.13994 & 65390 \\
-1.49856 & 60766 \\
-1.81629 & 36470\end{array}$ \\
\hline $\begin{array}{rr}6 g & \\
1.91114 & 56116 \\
1.65247 & 75486 \\
0.73068 & 20487 \\
0.14946 & 01872 \\
-1.46610 & 37437 \\
-1.97766 & 16525\end{array}$ & $\begin{array}{rl}6 h & \\
2.44475 & 93389 \\
2.11161 & 29670 \\
1.10878 & 13716 \\
-0.10878 & 13716 \\
-1.11161 & 29670 \\
-1.44475 & 93389\end{array}$ & $\begin{array}{rr}6 i \\
1.94985 & 58244 \\
1.56366 & 29649 \\
0.86776 & 74782 \\
-0.86776 & 74782 \\
-1.56366 & 29649 \\
-1.94985 & 58244\end{array}$ & $\begin{array}{rr}6 j & \\
2.12327 & 32553 \\
1.68547 & 18076 \\
0.68273 & 21397 \\
0.24385 & 63775 \\
-0.92932 & 81851 \\
-1.80600 & 53949\end{array}$ \\
\hline $\begin{array}{rr}6 k & \\
1.96961 & 55060 \\
1.28557 & 52194 \\
0.68404 & 02867 \\
-0.68404 & 02867 \\
-1.28557 & 52194 \\
-1.96961 & 55060\end{array}$ & $\begin{array}{rr}6 l \\
2.37360 & 03535 \\
2.07124 & 50231 \\
0.49785 & 73152 \\
-0.22710 & 87607 \\
-1.14649 & 15929 \\
-1.56910 & 23383\end{array}$ & \begin{tabular}{rr}
\multicolumn{2}{c}{$6 m$} \\
2.04670 & 58732 \\
1.38700 & 01779 \\
0.65570 & 47692 \\
-0.32801 & 78979 \\
-0.86250 & 94037 \\
-1.89888 & 35188
\end{tabular} & $\begin{array}{rr}6 n & \\
2.25517 & 13921 \\
1.85635 & 10103 \\
0.83422 & 15005 \\
0.12290 & 56033 \\
-1.37807 & 69953 \\
-1.69057 & 25109\end{array}$ \\
\hline 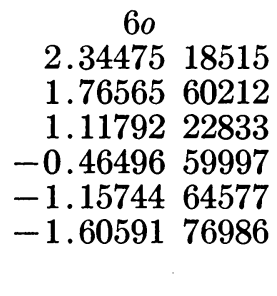 & 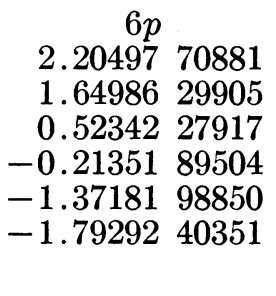 & $\begin{array}{rr}6 q \\
2.42486 & 15948 \\
1.75560 & 15024 \\
0.86345 & 85959 \\
-0.12887 & 98831 \\
-1.34132 & 18022 \\
-1.57372 & 00078\end{array}$ & \begin{tabular}{rr}
\multicolumn{2}{c}{$7 a$} \\
2.27880 & 70929 \\
1.93806 & 50941 \\
0.87572 & 24244 \\
0.24207 & 93268 \\
-0.57219 & 13441 \\
-1.17827 & 35857 \\
-1.58420 & 90085
\end{tabular} \\
\hline \begin{tabular}{rr}
\multicolumn{2}{c}{$7 b$} \\
2.39123 & 75594 \\
1.91325 & 34641 \\
1.23962 & 22465 \\
0.59378 & 69656 \\
-0.53556 & 36555 \\
-1.12898 & 87698 \\
-1.47334 & 78104
\end{tabular} & 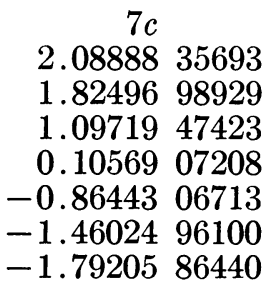 & $\begin{array}{rr}7 d \\
2.24120 & 40051 \\
1.70295 & 89324 \\
1.17760 & 79154 \\
0.25271 & 46462 \\
-0.40360 & 44121 \\
-1.32677 & 95062 \\
-1.64410 & 15808\end{array}$ & \begin{tabular}{rr}
\multicolumn{2}{c}{$7 e$} \\
2.15315 & 39967 \\
1.85310 & 01391 \\
1.14835 & 13343 \\
0.21950 & 92371 \\
-0.50960 & 73144 \\
-1.11551 & 11099 \\
-1.74899 & 62829
\end{tabular} \\
\hline
\end{tabular}


TABLE 2-Continued

\begin{tabular}{|c|c|c|c|}
\hline \begin{tabular}{rr}
\multicolumn{1}{c}{$7 f$} \\
2.20183 & 39730 \\
1.51066 & 11464 \\
0.80437 & 50613 \\
0.22934 & 60487 \\
-0.73300 & 93107 \\
-1.29073 & 24447 \\
-1.72247 & 44739
\end{tabular} & \begin{tabular}{rc}
\multicolumn{2}{c}{$7 g$} \\
2.53663 & 40001 \\
1.95021 & 60042 \\
1.13664 & 55841 \\
0.29238 & 53908 \\
-0.38268 & 33351 \\
-1.14473 & 53768 \\
-1.38846 & 22674
\end{tabular} & \begin{tabular}{rr}
\multicolumn{2}{c}{$7 h$} \\
2.18667 & 40539 \\
1.93346 & 91500 \\
0.93191 & 78785 \\
-0.13376 & 41384 \\
-0.78108 & 16535 \\
-1.39163 & 10759 \\
-1.74558 & 42146
\end{tabular} & \begin{tabular}{rr}
\multicolumn{2}{c}{$7 i$} \\
2.27555 & 45388 \\
1.84067 & 40073 \\
1.36914 & 49724 \\
0.68007 & 32224 \\
-0.43188 & 29664 \\
-1.07182 & 01568 \\
-1.66174 & 36178
\end{tabular} \\
\hline \begin{tabular}{rr}
\multicolumn{2}{c}{$7 j$} \\
2.07680 & 91667 \\
1.76493 & 74245 \\
1.27273 & 81755 \\
-0.10757 & 69753 \\
-0.79832 & 42651 \\
-1.32536 & 65502 \\
-1.88321 & 69762
\end{tabular} & 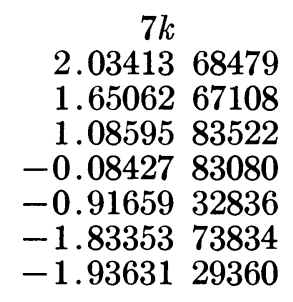 & \begin{tabular}{rr}
\multicolumn{2}{c}{$7 l$} \\
2.21007 & 56822 \\
1.87175 & 87733 \\
1.23180 & 73173 \\
0.66279 & 18687 \\
-0.88404 & 60243 \\
-1.33116 & 55726 \\
-1.76122 & 20446
\end{tabular} & \begin{tabular}{rr}
\multicolumn{2}{c}{$7 m$} \\
2.31441 & 11031 \\
1.68056 & 07022 \\
1.08337 & 53571 \\
-0.11845 & 28845 \\
-0.81025 & 58461 \\
-1.48862 & 73940 \\
-1.66101 & 10378
\end{tabular} \\
\hline \begin{tabular}{rr}
\multicolumn{2}{c}{$7 n$} \\
2.07804 & 57594 \\
1.81563 & 43448 \\
0.86539 & 15031 \\
0.23086 & 84170 \\
-0.41764 & 84063 \\
-1.66796 & 97214 \\
-1.90432 & 18966
\end{tabular} & \begin{tabular}{rr}
\multicolumn{2}{c}{70} \\
2.47747 & 11727 \\
2.05363 & 38895 \\
1.12041 & 46295 \\
0.25444 & 72868 \\
-0.53013 & 58647 \\
-0.85525 & 86034 \\
-1.52057 & 25103
\end{tabular} & \begin{tabular}{rr}
\multicolumn{2}{c}{$8 a$} \\
2.39886 & 13151 \\
2.15144 & 17932 \\
1.72978 & 03441 \\
0.89332 & 68549 \\
0.10667 & 31451 \\
-0.72978 & 03441 \\
-1.15144 & 17932 \\
-1.39886 & 13151
\end{tabular} & \begin{tabular}{rr}
\multicolumn{2}{c}{$8 b$} \\
1.96594 & 61994 \\
1.70043 & 42715 \\
1.20526 & 92728 \\
0.54732 & 59801 \\
-0.18453 & 67189 \\
-0.89147 & 67116 \\
-1.47801 & 78344 \\
-1.86494 & 44588
\end{tabular} \\
\hline $\begin{array}{rr}8 c & \\
2.45612 & 24152 \\
2.23131 & 98589 \\
1.52609 & 63417 \\
0.85105 & 97391 \\
0.14894 & 02609 \\
-0.52609 & 63417 \\
-1.23131 & 98589 \\
-1.45612 & 24152\end{array}$ & $\begin{array}{rr}8 d & \\
2.42876 & 01763 \\
2.05053 & 08962 \\
1.49093 & 45592 \\
0.63384 & 55510 \\
-0.15872 & 53449 \\
-0.73796 & 61606 \\
-1.21756 & 33176 \\
-1.48981 & 63597\end{array}$ & $\begin{array}{rr}8 e & \\
1.96157 & 05608 \\
1.66293 & 92246 \\
1.11114 & 04660 \\
0.39018 & 06440 \\
-0.39018 & 06440 \\
-1.11114 & 04660 \\
-1.66293 & 92246 \\
-1.96157 & 05608\end{array}$ & $\begin{array}{rr}8 f & \\
2.07451 & 94192 \\
1.69234 & 74357 \\
1.07758 & 22341 \\
0.29176 & 06409 \\
-0.52266 & 88171 \\
-1.19608 & 43121 \\
-1.56258 & 38332 \\
-1.85487 & 27675\end{array}$ \\
\hline $\begin{array}{rr}8 g \\
2.33390 & 51943 \\
2.06295 & 99589 \\
1.36617 & 23543 \\
0.79662 & 21523 \\
0.11468 & 99392 \\
-0.84795 & 88071 \\
-1.22662 & 28613 \\
-1.59976 & 79307\end{array}$ & $\begin{array}{rr}8 h \\
2.37877 & 05280 \\
1.92985 & 82731 \\
1.53431 & 37336 \\
0.68111 & 13800 \\
0.13096 & 00598 \\
-0.76991 & 00007 \\
-1.32584 & 28197 \\
-1.55926 & 11542\end{array}$ & $\begin{array}{rr}8 i \\
2.23208 & 53685 \\
1.81608 & 09200 \\
1.26804 & 86993 \\
0.71743 & 97852 \\
-0.15414 & 60600 \\
-0.70002 & 37500 \\
-1.47011 & 58178 \\
-1.70936 & 91452\end{array}$ & $\begin{array}{rr}8 j & \\
2.28928 & 02351 \\
1.96017 & 22617 \\
1.23336 & 56728 \\
0.47533 & 09359 \\
-0.24726 & 15874 \\
-0.67083 & 22418 \\
-1.38279 & 04401 \\
-1.65726 & 48363\end{array}$ \\
\hline \begin{tabular}{rr}
\multicolumn{2}{c}{$8 k$} \\
1.97537 & 66812 \\
1.78201 & 30484 \\
0.90798 & 09995 \\
0.31286 & 89301 \\
-0.31286 & 89301 \\
-0.90798 & 09995 \\
-1.78201 & 30484 \\
-1.97537 & 66812
\end{tabular} & \begin{tabular}{rr}
\multicolumn{2}{c}{$8 l$} \\
2.38706 & 40662 \\
2.23910 & 60007 \\
1.80781 & 37653 \\
1.07846 & 71566 \\
0.07300 & 11789 \\
-0.58531 & 69585 \\
-1.43404 & 98300 \\
-1.56608 & 53792
\end{tabular} & 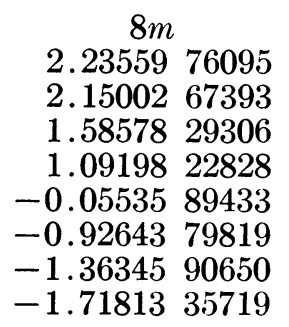 & $\begin{array}{rr}8 n & \\
2.15041 & 14940 \\
1.89964 & 35301 \\
1.36653 & 64300 \\
0.59805 & 20552 \\
-0.17581 & 47903 \\
-0.71891 & 58705 \\
-1.30764 & 92408 \\
-1.81226 & 36076\end{array}$ \\
\hline
\end{tabular}


TABLE 2-Continued

\begin{tabular}{|c|c|c|c|}
\hline $\begin{array}{rc}80 \\
2.22153 & 24876 \\
1.55561 & 23225 \\
1.16052 & 22500 \\
0.42908 & 59241 \\
-0.25745 & 81743 \\
-0.85931 & 74559 \\
-1.50656 & 25745 \\
-1.74341 & 47794\end{array}$ & $\begin{array}{rr}8 p \\
1.98288 & 97227 \\
1.58670 & 66806 \\
1.21752 & 28580 \\
0.26105 & 23844 \\
-0.26105 & 23844 \\
-1.21752 & 28580 \\
-1.58670 & 66806 \\
-1.98288 & 97227\end{array}$ & $\begin{array}{rr}8 q \\
2.29207 & 90117 \\
2.07909 & 08161 \\
1.43437 & 77844 \\
0.85052 & 13404 \\
-0.12637 & 03068 \\
-0.71552 & 88820 \\
-1.12851 & 51069 \\
-1.68565 & 46568\end{array}$ & \begin{tabular}{rr}
\multicolumn{2}{c}{$8 r$} \\
1.9890437907 \\
1.48628 & 96510 \\
0.81347 & 32862 \\
0.41582 & 33816 \\
-0.41582 & 33816 \\
-0.81347 & 32862 \\
-1.48628 & 96510 \\
-1.98904 & 37907
\end{tabular} \\
\hline \begin{tabular}{rc}
\multicolumn{2}{c}{$8 s$} \\
2.14606 & 73652 \\
1.71870 & 71300 \\
1.11900 & 47370 \\
0.36899 & 54337 \\
-0.31650 & 62081 \\
-0.81640 & 94247 \\
-1.38446 & 90891 \\
-1.83538 & 99441
\end{tabular} & $\begin{array}{rr}8 t \\
2.25891 & 73222 \\
2.05610 & 77585 \\
1.32533 & 98244 \\
0.87190 & 42270 \\
-0.40705 & 09516 \\
-0.89051 & 64126 \\
-1.49093 & 11702 \\
-1.72377 & 05976\end{array}$ & $\begin{array}{rr}8 u \\
2.27526 & 75277 \\
2.07518 & 51952 \\
1.66866 & 42589 \\
0.22173 & 55606 \\
-0.28125 & 07082 \\
-0.85062 & 92510 \\
-1.40016 & 57332 \\
-1.70880 & 68502\end{array}$ & $\begin{array}{rr}8 v & \\
2.28531 & 36141 \\
1.92850 & 54268 \\
1.59579 & 07216 \\
0.53854 & 47058 \\
-0.09757 & 05446 \\
-1.08935 & 57382 \\
-1.46075 & 51746 \\
-1.70047 & 30109\end{array}$ \\
\hline \begin{tabular}{rc}
\multicolumn{2}{c}{$8 w$} \\
2.23056 & 99297 \\
1.96804 & 19244 \\
1.08244 & 63149 \\
0.50902 & 52332 \\
-0.41744 & 54453 \\
-1.16793 & 35980 \\
-1.44876 & 59545 \\
-1.75593 & 84043
\end{tabular} & $\begin{array}{rr}8 x \\
2.38158 & 76097 \\
2.13059 & 68863 \\
1.64839 & 12780 \\
0.84150 & 09960 \\
0.21026 & 94526 \\
-0.32962 & 78318 \\
-1.27495 & 72375 \\
-1.60776 & 11534\end{array}$ & \begin{tabular}{rr}
\multicolumn{2}{c}{$8 y$} \\
2.29737 & 50522 \\
1.75862 & 05855 \\
0.90472 & 96661 \\
0.35035 & 54513 \\
-0.42535 & 04548 \\
-0.75418 & 45108 \\
-1.43342 & 86594 \\
-1.69811 & 71301
\end{tabular} & $\begin{array}{rr}8 z & \\
2.35172 & 16719 \\
2.04492 & 34889 \\
1.53038 & 81076 \\
0.45824 & 16131 \\
-0.44139 & 25348 \\
-0.83950 & 49209 \\
-1.45879 & 57700 \\
-1.64558 & 16558\end{array}$ \\
\hline
\end{tabular}

nomial. To take a specific case, we see from Table 1 that a sextic equation

$$
x^{6}+\cdots=0
$$

with integer coefficients and real roots, for which the span is less than 4 and the gap is less than 0.23 , is reducible. Indeed, there are just 17 essentially different irreducible sextics with span less than 4 , and the gaps vary from 0.6015 down to 0.2324 . On the other hand, there are many reducible sextics with span less than 4 having smaller gaps. Considering just the sextics which are the product of two different irreducible cubics, then our preliminary output showed that there are 19 inequivalent ones. (These could also be found by combining the five inequivalent cubics, and cubics equivalent to them, in all possible ways.) These reducible sextics fall into two groups: 7 with gaps from 0.5550 down to 0.3111 , which look no different from the irreducible sextics in this respect, and 12 with gaps from 0.1430 down to 0.0186 , whose gaps are too small for irreducible sextics. It would be interesting to find a general theorem relating the smallness of the gap between the nearest roots of a polynomial to its reducibility.

University of California

Berkeley, California 
1. L. Kronecker, "Zwei Sätze über Gleichungen mit ganzzahligen Coefficienten," $J$. Reine Angew. Math., v. 53, 1857, p. 173-175.

2. EMma T. LEHMER, "A numerical function applied to cyclotomy," Bull. Amer. Math. Soc., v. 36, 1930, p. 291-298.

3. R. M. ROBINSON, "Intervals containing infinitely many sets of conjugate algebraic integers," Studies in Mathematical Analysis and Related Topics: Essays in Honor of George Pólya, Stanford, 1962, p. 305-315.

4. R. M. RoBInson, "On the spans of derivatives of polynomials," Amer. Math. Monthly, v. 71,1964, p. 504-508. 